\title{
Doing Age in Kulturorganisationen - Ein praxeologischer Blick auf Altersbilder in der kulturellen Bildung
}

\author{
Vera Gallistl · Viktoria Parisot · Julia Birke
}

(C) Der/die Autor(en) 2019

Zusammenfassung Mit dem demografischen Wandel verlängert sich nicht nur die Lebenszeit von Individuen, es findet auch eine Veränderung der Lebensphase Alter selbst statt. Statt einem lange vorherrschenden Ruhestandsmodell zu folgen, fokussieren Politik und Wissenschaft zunehmend auf die Aktivierung der älteren Bevölkerung. Bildung, Lernen und kulturelles Engagement älterer Menschen gewinnen damit an Bedeutung. Gleichzeitig stellt die kulturelle Bildung für Menschen in der nachberuflichen Phase ein Randthema dar, das bislang kaum öffentlich diskutiert oder wissenschaftlich erforscht wurde. Während für ältere Menschen also zunehmend der Anspruch entsteht, ihre nachberufliche Lebensphase aktiv zu gestalten, finden sich in Bildungs- und Kulturorganisationen bislang selten Angebote, in denen diese Ansprüche realisiert werden können.

Ausgehend von Teilergebnissen des Projekts ,Zugangsbarrieren für ältere Menschen in der kulturellen Bildung - ,Mainstreaming Ageing ' im Kultursektor“ (2016-2018) wird im folgenden Beitrag basierend auf sechs qualitativen Fallstudien kultureller Bildungsangebote für ältere Menschen der Frage nachgegangen, welche impliziten Altersbilder sich in kulturellen Bildungsangeboten in Österreich finden. Dabei wird ein Schwerpunkt darauf gelegt, wie diese in der Angebotsgestaltung diskursiv verhandelt werden, wie sich diese durch Lernziele ausdrücken und welche sozialen Positionen dabei für ältere Teilnehmende entstehen. Aufbauend darauf wird der Frage nachgegangen, welche Rolle Selbstbestimmung, Selbstorganisation und Empowerment älterer Menschen in kulturellen Bildungsangeboten spielen und wie

\footnotetext{
V. Gallistl $(\bowtie) \cdot$ V. Parisot $\cdot$ J. Birke

Institut für Soziologie, Universität Wien, Rooseveltplatz 2, 1090 Wien, Österreich

E-Mail: vera.maria.gallistl@univie.ac.at

V. Parisot

E-Mail: viktoria.parisot@univie.ac.at

J. Birke

E-Mail: julia.birke@univie.ac.at
} 
intergenerationelle Ansätze und Kreativität Selbstbestimmung im Alter unterstützen können.

Abschließend plädieren wir im vorliegenden Beitrag dafür, kulturelle Bildung für Ältere im Sinne einer praxeologischen Perspektive zu betrachten und das Augenmerk vor allem auf den Herstellungsprozess zu legen, in dem die Bildungsangebote von verschiedenen AkteurInnen getan und ausagiert werden. (Kulturelle) Bildung im Alter ist - wie in anderen Lebensphasen auch - als ein spezifischer Prozess der Subjektwerdung und Sozialisation zu verstehen, in dem ältere Menschen lernen, sich gemäß ihrem Alter zu verhalten. Einen kritischen Blick auf die impliziten Bilder und Vorstellungen über das Alter(n) in diesem Prozess zu richten, kann dazu beitragen, blinde Flecken bestehender Angebote zu identifizieren und altersinklusive Angebote der kulturellen Bildung zu schaffen, sodass „Mainstreaming Ageing“ im Kultursektor möglich wird.

Schlüsselwörter Age $\cdot$ Ageing $\cdot$ Altersbilder $\cdot$ Praxistheorien · Lebensbegleitendes Lernen

\section{Doing age in cultural organizations-a praxeological approach towards ageing in cultural education}

Abstract Demographic change not only prolongs life expectancy of individuals, it also changes the meaning of age and ageing itself. While old age was long characterized as a life-stage of social disengagement and health decline, European policies increasingly put an emphasis on active ageing, promoting the social and cultural engagement of older adults. Lifelong learning and cultural engagement become cornerstones of active ageing imperatives. However, cultural education for older adults as a means to support cultural engagement in later life has hardly been researched or publicly discussed. While older adults are increasingly expected to engage in learning in later life, educational and cultural organizations hardly attend to this expectation.

This paper presents results of the project "Barriers to Cultural Education in Old Age-'Mainstreaming Ageing' in Cultural Organizations (2016-2018)". Based on six case studies in cultural education programs for older adults, we examine which implicit images of ageing are conveyed through these programs. Using situational analysis and a doing-perspective on age and ageing, we explore how images of ageing and the social positions of older participants within the programs are negotiated. Further, we ask how such programs could serve as a means of empowerment in old age and which role cultural engagement and creativity play in older adults' empowerment.

Results indicate that research on cultural education for older adults could benefit from a practice-theoretical approach, as it allows researchers to explore how images of ageing are set into practice through various actors in cultural organizations. Cultural education in old age is a field of socialization and subjectification, in which older adults learn how to behave according to their age. Implementing "mainstreaming ageing" in cultural organizations in order to establish more age- 
inclusive programs and initiatives calls for a critical reflection on which images and ideas of ageing are negotiated through existing programs.

Keywords Age · Ageing · Doing Age · Practice Theories · Lifelong Learning

\section{Einleitung: ,Doing Age“ - Alter(n) und soziale Praxis}

Der demografische Wandel führt dazu, dass sich die Altersstruktur westlicher Gesellschaften grundlegend verändert. In den letzten 160 Jahren ist die Lebenserwartung stetig gestiegen (Buttler 2003) und Prognosen gehen davon aus, dass sich dieser Trend auch in Zukunft fortsetzen wird (Birg und Flöthmann 2002; Kontis et al. 2017). Durch die Zunahme der Lebenserwartung bei gleichzeitiger Stagnation der Fertilitätsrate kommt es zu einem Anstieg des Anteils älterer Menschen in allen europäischen Gesellschaften. In Österreich lag der Anteil an Menschen im Alter von 65 Jahren oder älter im Jahr 2017 bei 18\%, bis zum Jahr 2030 wird er voraussichtlich auf $23 \%$ steigen (Statistik Austria 2018).

Diese Entwicklungen machen einen soziologischen Blick auf die Lebensphase Alter notwendig. Das Alter(n) wurde - sowohl in der öffentlichen Wahrnehmung als auch in der Alterssoziologie bzw. Gerontologie - lange als ein biologischer Determinismus verstanden, der durch die Abnahme der kognitiven und körperlichen Fähigkeiten gekennzeichnet ist. Damit einhergehend, so die lange verbreitete Annahme, verändern sich auch die sozialen Rollen älterer Menschen im höheren Lebensalter hin zu mehr Rückzug und Inaktivität (vgl. Disengagement-Theorie, Cumming und Henry 1961). In den 1990er-Jahren hat sich der wissenschaftliche und öffentliche Blick auf die Lebensphase Alter zunehmend gewandelt. Ideen des aktiven Alterns fanden Eingang in die wissenschaftliche Diskussion (vgl. ActivitiyTheory, Havighurst 1961) und den politischen Diskurs (vgl. hierzu das Jahr des aktiven Alterns der Europäischen Kommission 2012, COM 2014). Als Gegenmodell zur Disengagement-These wurde hier in Richtung einer Aktivierung der älteren Bevölkerung argumentiert: Wer in sozialen Zusammenhängen aktiv bleibt, hat bis ins hohe Alter Chancen auf soziale Eingebundenheit und eine hohe Lebensqualität. So verweist die Forschung auf eine positive Wirkung von aktiver Teilhabe an Bildungsangeboten (Jenkins und Mostafa 2015) und ehrenamtlichem Engagement (Litwin und Stoeckel, 2013) auf die Lebensqualität und Gesundheit im Alter. Als besonders wirksam in diesem Zusammenhang wurden immer wieder kreative Tätigkeiten beschrieben (vgl. etwa für Review-Studien Bernard und Rickett 2016; Fraser et al. 2015).

In der jüngeren alterssoziologischen Diskussion wurden beide Perspektiven aufgrund ihrer (mehr oder weniger) versteckten Normativität zunehmend kritisiert (Amann und Kolland 2014) und der Fokus verlagerte sich stärker auf die Frage, durch welche Prozesse das soziale Alter hergestellt und verhandelt wird (Schroeter 2009). Forschungsergebnisse haben hier gezeigt, dass das Anzeigen von Alterskategorien als strukturierendes Element des Alltags verstanden werden kann, das sich etwa anhand einer bestimmten Form, sich zu kleiden (Twigg 2015), oder in der Art und Weise, wie öffentliche Räume genutzt werden (Wanka 2018), untersuchen 
lässt. Alter(n) ist vor diesem Hintergrund nicht als biologische Tatsache, sondern als fortlaufender Teil sozialer Praxis zu verstehen, an der viele unterschiedliche (menschliche und nicht-menschliche) AkteurInnen beteiligt sind. Das Alter(n) als soziologischen Tatbestand zu untersuchen, bedeutet vor dem Hintergrund einer kulturellen Gerontologie (Twigg und Martin 2014) bzw. eines „Doing Age“-Ansatzes (Schroeter 2009), Alter(n) als eingebettet in Sinn- und Bedeutungszuschreibungen und als Teil einer alltäglichen sozialen Praxis zu verstehen. Alter(n) zu untersuchen heißt damit, den Fokus auf ,die Herstellung (Konstruktion) von Altersdifferenzen [...], die nicht natürlich oder biologisch sind, die jedoch, sobald sie einmal konstruiert sind, wie real existierende Tatbestände behandelt werden“ (Schroeter 2009, S. 360), $\mathrm{zu}$ legen.

Wer und was ist aus so einer Perspektive an Prozessen des Älterwerdens beteiligt? Hier werden praxistheoretische Grundlagen relevant, die eine soziale Praxis als „Nexus of Doings and Sayings“ (Schatzki 2014, S. 18) verstehen. Soziale Praktiken sind geteilte Handlungs- und Denkvollzüge, in denen Menschen, Dinge und Diskurse in der konkreten Praxis des Tuns verbunden werden. Soziale Praktiken sind damit einerseits immer ein Tun-Mit - indem mehrere AkteurInnen miteinander in der Praxis verbunden werden - und ein Werden-Durch - in dem Sinne, dass Subjektivierungsformen durch Praktiken entstehen können (Reckwitz 2003). Das Älterwerden - sich selbst als eine alte Person wahrzunehmen - kann aus dieser Perspektive als ein Resultat sozialer Praxis verstanden werden.

Bildungsangebote für ältere Menschen können in diesem Prozess eine zentrale Rolle spielen, weil sie TeilnehmerInnen als ältere Menschen adressieren und so altersbezogene Subjektivierungsformen ein Teil des Lernens im Angebot werden. Eine Auseinandersetzung mit dem eigenen Alter(n) ist damit inhärenter Teil nachberuflicher Bildung (Schramek 2018). Durch die Teilnahme an Bildung in der nachberuflichen Phase lernen TeilnehmerInnen also nicht nur neue Kompetenzen, sondern auch, sich selbst als ältere TeilnehmerIn im Angebot wahrzunehmen. Bildung in der nachberuflichen Phase wird damit Teil eines „Doing Age“, weil es eine Auseinandersetzung mit dem eigenen Alter anstoßen kann (Gallistl et al. 2019).

Vor diesem Hintergrund stellt sich die Frage, wie das Alter(n) der TeilnehmerInnen in Angeboten kultureller Bildung zum Thema gemacht wird: Welches Alter(n) wird in den Angeboten thematisiert und erlernt? Wie ordnen sich die erlernten Altersbilder in der kulturellen Bildung in die oben skizzierte Dichotomie zwischen Aktivitäts- und Disengagementorientierung des Alter(n)s ein? Diese Forschungsnotiz möchte einen Beitrag zur Beantwortung dieser Fragen leisten, indem die folgenden Themen bearbeitet werden:

- Wie werden ältere Menschen von kulturellen Bildungsangeboten adressiert? Welche Annahmen über das Alter(n) sind damit verbunden?

- Welche Rollen, Positionen und Eigenschaften werden älteren TeilnehmerInnen damit in kulturellen Bildungsangeboten zugeschrieben?

- Welche Wege gelingender Integration älterer Menschen in die kulturelle Bildung können auf Basis dieser Erkenntnisse aufgezeigt werden? 


\subsection{Kulturelle Bildung für ältere Menschen}

Die positiven Wirkungen von künstlerischen und kulturellen Tätigkeiten auf die Lebensqualität (Bernard und Rickett 2016; Fraser et al. 2015), die Gesundheit (Noice und Noice 2013) oder die Entwicklung von positiven Einstellungen gegenüber dem Älterwerden (Reynolds 2015) wurden immer wieder hervorgehoben, trotzdem stellen ältere Menschen eine unterrepräsentierte Zielgruppe der kulturellen Bildung dar (De Groote und Neubauer 2009). Ebenso zeigt sich, dass sich Bildungschancen mit dem Austritt aus dem Erwerbsleben verändern: Die Wahrscheinlichkeit für eine Teilnahme an Bildungsangeboten sinkt in der nachberuflichen Phase (Gallistl und Wanka 2016).

Der vorliegende Beitrag beschäftigt sich ausgehend von diesen Befunden mit der Frage, wie ältere Menschen von der kulturellen Bildung in Österreich adressiert werden und welche Strategien empfohlen werden können, um ältere Menschen stärker zu integrieren. Wird im Folgenden von „kultureller Bildung“ gesprochen, so meint dies einerseits „education in the arts“ als auch „education through the arts“ (Bamford 2009, S. 12). Bei „Bildung in den Künsten“ können in kulturpädagogischen Angeboten Wissen und Fertigkeiten zum Verständnis und zur Ausübung künstlerisch-kreativer Aktivitäten erworben und kulturelle Identität entwickelt werden (Zembylas 2007; Bamford 2009). Bei „Bildung durch die Künste“ können durch die Beschäftigung mit Kunst und Kultur Lern- und Auseinandersetzungsprozesse des Menschen mit sich, seinen Mitmenschen und der Gesellschaft stattfinden (Zembylas 2007; Dallmann et al. 2008).

\section{Methodische Herangehensweise}

Grundlage der hier dargestellten Teilergebnisse sind sechs qualitative Fallstudien in kulturellen Bildungsangeboten, die im Rahmen des Forschungsprojektes „Zugangsbarrieren für ältere Menschen in der kulturellen Bildung - ,Mainstreaming Ageing ' im Kultursektor" (gefördert durch den Jubiläumsfonds der ÖNB, 2016-2018) ${ }^{1}$ durchgeführt wurden. Zur Untersuchung herangezogen wurden sechs Beispiele aus den Bereichen Tanz, Theater, Musik, Literatur, bildende Kunst und Technik vor dem Hintergrund maximaler Kontrastierung: Auf Basis einer Online-Umfrage wurden zunächst österreichweit Angebote der kulturellen Bildung für ältere Menschen gesammelt und danach auf Basis größtmöglicher Heterogenität hinsichtlich der Regionalität (Bundesland), der adressierten Zielgruppe (altershomogen/intergenerationell), der Häufigkeit (einmalig/regelmäßig) und der Kultursparte, zur weiteren Untersuchung ausgewählt. In jedem dieser Angebote wurde eine qualitative Fallstudie durchge-

\footnotetext{
${ }^{1}$ Das Forschungsprojekt umfasst insgesamt vier methodische Schritte: 1) Dokumentation von kulturellen Bildungsangeboten für ältere Menschen in Österreich auf Basis einer Online-Umfrage, 2) GovernanceAnalyse des Feldes der kulturellen SeniorInnenbildung auf Basis von sechs qualitativen Fallstudien in Kulturorganisationen, 3) Potenzial-Analyse auf Basis von sechs qualitativen Fallstudien in kulturellen Bildungsangeboten für ältere Menschen und 4) Entwicklung von Leitlinien zum „Mainstreaming Ageing“ im Kultursektor. Die im Rahmen dieser Forschungsnotiz vorgestellten Ergebnisse beziehen sich auf die Ergebnisse der dritten Forschungsphase.
} 
Tab. 1 Übersicht der untersuchten kulturellen Bildungsprojekte

\begin{tabular}{lll}
\hline Art des Bildungsangebots & Angegebene Zielgruppe & Dauer \\
\hline Tanzkurs & SeniorInnen & Wöchentlich, 90min \\
Jodelseminar & Nicht näher spezifiziert & Zweitägiges Wochenendseminar \\
Smartphone-Kurs & Über 55 Jahre & Drei Einheiten, je 90min \\
\hline
\end{tabular}

führt: Es wurden zum einen episodische Interviews mit Teilnehmenden (Flick 2011; zit. nach Misoch 2015), zum anderen problemzentrierte Interviews (Witzel 2000) mit den Kulturvermittelnden geführt. Zusätzlich nahmen an jedem Angebot mindestens zwei ForscherInnen teil und erstellten Beobachtungsprotokolle.

Zur Datenauswertung wurden alle Daten mithilfe von Max QDA 2018 kodiert. Der Datenkorpus wurde mittels Situationsanalyse (Clarke 2012) zyklisch analysiert. Situationsanalysen eignen sich besonders als Auswertungsmethode praxeologischer Forschung (Both 2015), es wird dabei die Forschungssituation selbst als Hauptuntersuchungseinheit in den Mittelpunkt gestellt. In der Auswertung wurden dabei u. a. menschliche (z.B. TeilnehmerInnen), nicht-menschliche (z. B. die Räume, in denen ein Angebot stattfand) und diskursive (z. B. Normativitäten rund um aktives Altern) Elemente berücksichtigt. In Anlehnung an Geertz' (2007) ,dichte Beschreibungen“ wurden „,dichte Analysen“ für jedes Angebot getrennt erstellt, darauf aufbauend wurden Gemeinsamkeiten und Unterschiede der einzelnen Bildungsangebote interpretiert.

Die hier dargestellten Ergebnisse beziehen sich auf die gemeinsame Analyse der Daten der sechs erhobenen Fallstudien. Zugunsten einer besseren Nachvollziehbarkeit werden für die Ergebnisdarstellung in diesem Beitrag drei der sechs untersuchten Angebote herangezogen, diese sind in Tab. 1 näher beschrieben. Die hier nicht ausgeführten Angebote - ein Theaterworkshop, ein Literaturworkshop und eine Museumsführung für demenzerkrankte Menschen - wurden in Hinblick auf die Kontrastierung der Fälle (Glaser und Strauss 2010) aus der Ergebnisdarstellung ausgeschlossen. Die zentralen Ergebnisse dieser hier nicht dargestellten Fallstudien decken sich allerdings mit den ausgeführten Zusammenhängen.

Um von einer deskriptiven auf eine abstrahierte Ebene zu gelangen, wurden die Daten im Zuge einer rekonstruktiven Auswertung analysiert und die Fallstudien miteinander verschränkt ausgewertet. Durch kontrastierende Fallauswahl sowie Triangulation verschiedener Methoden wurde dabei die interne Validität der Daten erhöht. Im Zuge dessen konnte eine Generalisierbarkeit der Ergebnisse in Form kontextspezifischer Aussagen (Mayring 2007) erreicht werden. Angemerkt wird, dass die dargestellten Ergebnisse aufgrund ihrer Kontext- und Situationsgebundenheit nicht ohne Weiteres auf andere Forschungsgegenstände übertragen werden können.

In der folgenden Ergebnisdarstellung möchten wir den Fokus nun auf zwei zentrale Elemente der Situation legen, durch die das Alter(n) in den untersuchten Angeboten Relevanz erhielt: Einerseits besprechen wir die diskursiven Vorstellungen über das Alter(n), die in der Angebotsgestaltung und den Zielsetzungen der Angebote zum Tragen kamen, andererseits beschreiben wir deren Relevanz in Bezug auf Subjektivierungsprozesse und soziale Positionen älterer Menschen in Kulturorganisationen. Abschließend diskutieren wir auf Basis dieser Forschungsergebnisse, inwiefern 
Selbstermächtigungsstrategien für ältere Menschen durch kulturelle Bildung angestoßen wurden und wie diese von zukünftigen Angeboten verstärkt berücksichtigt werden können.

\section{Ergebnisse}

Die Ergebnisse werden in zwei Abschnitten dargestellt. Zunächst wird darauf eingegangen, welche Altersbilder sich in den untersuchten kulturellen Bildungsangeboten fanden. Im Analyseprozess zeigte sich hier deutlich, dass die Bildungsangebote von verschiedenen Vorstellungen des Alter(n)s geprägt waren - so wurden älteren Menschen in den Angeboten spezifische Eigenschaften zugeschrieben und Ziele der Angebote vor dem Hintergrund dieser spezifischen Vorstellungen über das Älterwerden festgelegt. Zweitens zeigten die Daten, dass diese Formulierung von Zielvorstellungen in der Arbeit mit älteren Menschen als Subjektivierungsprozesse verstanden werden können, die soziale Positionen für ältere Menschen in den Bildungsangeboten festschrieben.

\subsection{Altersbilder in den Zielsetzungen kultureller Bildungsangebote}

Es zeigte sich, dass die Zielgruppe älterer Menschen in den Angeboten großteils als schützenswerte Gruppe konzipiert wurde. Die untersuchten Angebote orientierten sich - häufig eher implizit als explizit - an der Vorstellung, dass ältere Menschen weniger leistungsfähig als jüngere sind. Die Vorstellung von der geringeren Leistungsfähigkeit älterer Menschen wurde mit altersbedingten körperlichen Abbauprozessen begründet. Ziele der untersuchten Angebote waren deswegen häufig eher der Erhalt von Kompetenzen statt deren Auf- und Ausbau. Dieses Rollenverständnis von älteren Menschen als Nicht-Professionelle schrieb ihnen in Kulturorganisationen eine randständige Position zu (siehe 3.2.).

Im Tanzangebot wurde die Konzeption von älteren Menschen als schützenswerte Zielgruppe besonders deutlich. Es handelte sich hierbei um ein wöchentlich stattfindendes Angebot, bei dem sich eine Gruppe von etwa 40 älteren Menschen überwiegend Frauen über 60 Jahre - in einer Sporthalle zum gemeinsamen Tanz traf. Die einzelnen Tänze wurden von der Kulturvermittlerin vorgegeben, wie eine Forscherin in einem Beobachtungsprotokoll beschrieb:

Der Ablauf ist immer so, dass die Kulturvermittlerin die Schrittfolge zeigt, dann werden sie ohne Musik trockengeübt und anschließend wird ein ganzes Stück lang die Choreografie getanzt (Beobachtungsprotokoll 1, Tanzangebot).

Ziel des Angebots war laut Kulturvermittlerin, dass die TeilnehmerInnen trotz des steigenden Alters bestimmte Tanzpraktiken erlernen konnten. Zwar seien ältere Menschen in Bezug auf ihre Bewegungsfähigkeiten eingeschränkt, doch könnte durch die spezielle Ausrichtung des Angebots erreicht werden, dass das Erlernen von Tänzen bis ins hohe Alter möglich ist. Die Zielsetzungen des Angebots beschrieb sie so: 
Ich bin alt. Ich kann also verschiedene Dinge nicht mehr machen. Aber ich habe noch die Chance, anderes zu machen. Wirklich eben etwas, was meinem Alter entspricht (Kulturvermittlerin, Tanzangebot).

Welche impliziten Vorstellungen über das Alter wurden damit in diesem Angebot verhandelt? Hier zeigte die Analyse der Daten, dass die Zielsetzungen des Angebots vor allem von einem biologischen Altersbild geprägt waren, welches die Abnahme körperlicher Leistungsfähigkeit im Alter betonte. Vor dieser Annahme der grundsätzlichen biologischen Einschränkung älterer Menschen zielte das Angebot vor allem auf die körperliche Aktivierung der älteren Zielgruppe:

Und gerade für ältere Menschen ist ja die Sturzgefahr sehr groß. Und das ist durch dieses Tanzen sehr oft, dass diese Sicherheit, diese Trittsicherheit oder die Standfestigkeit, dass das dann alles ein bisschen gestärkt wird oder geschult wird (Kulturvermittlerin, Tanzangebot).

Verschiedene Sportarten und Bewegungsmöglichkeiten wurden in den Interviews klar jüngeren oder älteren Menschen zugeschrieben: Körperliche Betätigung wurde damit vor der Kontrastfolie ,jung vs. alt“"interpretiert. Das besuchte Angebot wurde als Sportart gesehen, die für ältere Personen geeignet ist. Als Voraussetzung für eine Teilnahme an dem Tanzangebot wurde die Identifikation mit einem Altersbild beschrieben, welches primär körperliche Einschränkungen verhandelt. So beschrieb etwa eine Teilnehmerin, dass eine Freundin nie an dem Angebot teilnehmen würde, weil sie dafür noch zu ,jung“ - d.h. körperlich leistungsfähig - sei:

Meine Freundin [...] die ist im gleichen Alter wie ich, die würde zum Beispiel nie zum [Tanzangebot] gehen. Weil sie nämlich von Haus aus sehr viel beweglicher ist und mehr [die] Sportarten von Jüngeren macht (Teilnehmerin (61), Tanzangebot).

Die Zielsetzung des Angebots wurde also unter der Annahme verhandelt, dass die Teilnehmenden mit körperlichen Einschränkungen konfrontiert sind, die als mit dem Lebensalter verbunden interpretiert wurden. Alter(n) wurde damit in den Angeboten vor allem als ein biologischer Prozess gedeutet.

\subsection{Soziale Positionen älterer Menschen in Kulturorganisationen}

Die in den Angeboten verhandelten Altersbilder und die daraus hervorgehenden Strategien in den Lernzielen der Angebote produzierten verschiedene soziale Positionen für ältere Menschen im Lernprozess und in Kulturorganisationen. Im Wesentlichen wurden zwei Positionen sichtbar, die sich an der Dichotomie der Lehrenden und Lernenden orientierten.

Ältere Menschen als weniger wissend und körperlich leistungsfähig zu verstehen, bedeutete im Lernprozess, dass eine deutliche Asymmetrie zwischen den Lehrenden und den älteren TeilnehmerInnen erkennbar wurde. Dies äußerte sich besonders im untersuchten Smartphone-Kurs. Hierbei handelte es sich um einen mehrteiligen Kurs, der für Personen über 55 Jahren konzipiert wurde. Mittels Frontalunterricht wurden Basiskenntnisse im Umgang mit Smartphones vermittelt: Zunächst zeigte die 
Trainerin eine Übung auf ihrem Smartphone auf einer Leinwand vor, anschließend konnten die TeilnehmerInnen die Übung auf ihrem eigenen Gerät durchführen (bei Bedarf mit Unterstützung der Trainerin). Nachdem keine Lernunterlagen ausgegeben wurden, war der Lernfortschritt für die Beteiligten wesentlich davon abhängig, im Angebot möglichst nahe an den vorgezeigten Schritten der Trainerin zu bleiben, wie sich auch für eine der Forscherinnen während des Kurses zeigte:

Ich versuche, auf meinem Handy mitzumachen [...] Obwohl die Dinge, die sie vorzeigt, irgendwie banal auf mich wirken, finde ich es schwierig, dabei zu bleiben (Beobachtungsprotokoll 1, Smartphone-Kurs).

„Dabei“ zu bleiben und die Schritte mitmachen zu können, zeigte sich durch die Struktur des Angebots als wesentlich für den Lernfortschritt.

In der Analyse des Angebots wurde deutlich, dass ältere Menschen als technikfern gesehen wurden und sich auch - mit Verweis auf den Vergleich mit jüngeren Altersgruppen - selbst so wahrnahmen.

Weil es ist halt so, viele haben Kinder oder irgendwelche Jüngeren wo man hingehen kann, und sagen, könntest du mir das bitte erklären? Und wenn du da keinen hast, dass du auch in den Kurs hingehen kannst (Teilnehmerin (54), Smartphone-Kurs).

Das „Nicht-Wissen“ in Bezug auf digitale Technologien war ein wesentlicher Bestandteil der Selbst- und Fremdbeschreibungen der Teilnehmenden. So führte die Kulturvermittlerin als zentralen positiven Aspekt des Angebots aus:

Dieses Bewusstsein, du bist nicht alleine mit [...] deinem Nicht-Wissen, sondern es gibt noch genug andere Leute, denen es auch so geht (Trainerin, Smartphone-Kurs).

Dass die Trainerin im Angebot als einzige Wissensressource zur Verfügung stand und auch keine Konzepte zur Anwendung kamen, in denen sich die Teilnehmenden wechselseitig im Lernprozess unterstützen hätten können, verstärkte die Asymmetrie zwischen den diskursiven Figuren der ,wissenden Lehrenden“ bzw. den als technikfern gesehenen ,älteren Lernenden“. Daraus entstehende Abhängigkeitsverhältnisse fanden durch das Bildungsangebot keine Auflösung, da wenig selbstermächtigende Aspekte - im Sinne eines Hinführens zu einem selbstorganisierten und selbstbestimmten Lernen (Bubolz-Lutz et al. 2010) - umgesetzt werden konnten.

Gegensätzlich dazu zeigte sich ein Bildungsangebot aus der Sparte Gesang, in dem die älteren Teilnehmenden als Lehrende verstanden wurden. Das Jodelseminar, das an einem Wochenende in den Bergen stattfand, war ohne Fokus auf spezifische Altersgruppen konzipiert. Überwiegend wurde es von älteren Frauen über 60 Jahren besucht, einige wenige TeilnehmerInnen waren unter 30 Jahre alt. Das Altersbild, dem das Angebot folgte, beschrieb ältere Menschen als erfahren, ruhig und zurückgezogen, wie im folgenden Zitat angedeutet:

Altern heißt, dass man reich an Jahren wird, vielleicht an Erfahrung. Ja, an Erfahrungen, Begegnungen, dass man vielleicht im Alter - das ist jetzt ein Detail - ein bisschen gütiger wird, ein bisschen vielleicht mehr nachdenkt immer, nicht mehr mit dem Kopf durch die Wand (Kulturvermittler, Jodelseminar). 
In dieses Altersbild eingebettet war die Vorstellung, dass die älteren TeilnehmerInnen semi-professionelle ExpertInnen für die Tradition des Jodelns darstellten. Weil Jodeln als altes Kulturgut beschrieben wurde, das primär von der älteren Generation tradiert wird, hatten die älteren Teilnehmenden die Rolle von Lehrenden, die - den professionellen Kulturvermittler unterstützend - jüngeren Teilnehmenden Tradition und Wissen weitergeben sollten. Dies wurde auch in den Selbstbeschreibungen der Teilnehmenden deutlich. So beschrieb etwa eine Teilnehmerin, dass die Weitergabe ihres Wissens an die nächste Generation eine wichtige Motivation für ihre Teilnahme am Angebot darstellte:

Ich bin ja schon alt und ich will schauen, dass Leute nachkommen, dass wieder Jüngere dabei sind und dass sie das einmal erleben dürfen (Teilnehmerin, Jodelseminar).

Die Teilnehmerin, die im Zitat andeutete ,schon alt“ $\mathrm{zu}$ sein und daher Jüngere für das Jodeln begeistern will, beschrieb hier implizit ein Alter, das perspektivisch mit Rückzug und einem Nicht-mehr-Können verbunden war. Dieser Rückzug sprach aber der aktiven Rolle älterer Menschen im Angebot nicht entgegen, sondern äußerte sich im Auftrag, das Wissen an die nächsten Generationen weiterzugeben. Auch hier zeigte sich, dass durch das vermittelte Altersbild im Angebot eine spezifische Position für ältere Menschen geschaffen wurde: Zwar wurden die älteren TeilnehmerInnen hier nicht als weniger kompetent als (jüngere) Lehrende adressiert, doch wurde als eine deutliche Rollenerwartung an ältere Menschen erkennbar, hohe Kompetenzen im Umgang mit Volksmusik zu besitzen. Auch daraus ergibt sich aufgrund äußerer Zuschreibungen eine bestimmte Rolle älterer Menschen in der kulturellen Bildung.

\section{Handreichungen an die kulturelle Bildung}

Die hier skizzierten Ergebnisse des Forschungsprojektes machten deutlich, dass ältere Menschen zwar häufig als spezielle Zielgruppe der kulturellen Bildung konzipiert wurden, diese Konzeption sich allerdings oft der Vorstellung von Alter(n) als biologischem und defizitärem Abbauprozess bediente. Aus gerontologischer Perspektive ist dieser Befund bedeutsam, da so Potenziale des Älterwerdens - wie Befreiung durch das Austreten aus dem Erwerbsleben oder gesteigertes soziales und politisches Engagement - von der kulturellen Bildung kaum adressiert wurden. Die Ergebnisse zeigen damit auf, wie negative Altersbilder, die in westlichen Gesellschaften die Norm darstellen (Gulette 2004), durch die Art und Weise, wie ältere Menschen von Kultur- und Bildungsorganisationen adressiert wurden, realisiert und stabilisiert wurden.

Welche Empfehlungen an die AnbieterInnen kultureller Bildung lassen diese Forschungsergebnisse zu? Die Daten machten deutlich, dass Selbstermächtigung bzw. Empowerment für ältere Menschen in den Angeboten eine untergeordnete Rolle spielten: Statt der Ausweitung von Handlungsmöglichkeiten zielten die untersuchten Projekte vor allem auf den Erhalt von Kompetenzen ab. Trotzdem wurde in den Angeboten deutlich, dass gerade kulturelle Bildung als Möglichkeitsraum gesehen werden kann, in dem Potenzial zur Selbstermächtigung älterer Menschen steckt. 
Entsprechend verweisen wir auf drei Aspekte, die zukünftig in der Gestaltung kultureller Bildungsangebote für ältere Menschen berücksichtigt werden können:

Erstens war Altern in den meisten Angeboten zwar eine implizite Kategorie, trotzdem wurde in den Interviews immer wieder darauf verwiesen, dass das Entdecken der eigenen kreativen Potenziale als Strategie funktionieren könne, den Status des „Alt-Seins“ - welcher häufig negativ konnotiert war - um Facetten der eigenen Persönlichkeit zu erweitern bzw. als Kategorie aufzuweichen. Im Analyseprozess wurde deutlich, dass positive Diskurselemente zu Altern - die auch Gestaltungsmöglichkeiten eröffneten - erst durch die explizite Thematisierung des Alterns möglich wurden. Kulturelle Bildung hat damit das Potenzial, das Alter(n) zum Thema der Bildung $\mathrm{zu}$ machen und Alternativen zu einem defizitorientierten Altersbild zu entwerfen (Baumann und De Groote 2018).

Die explizite Thematisierung des Alterns erfolgte zweitens besonders in intergenerationellen Angeboten. Durch das generationenübergreifende Setting wurde erreicht, Unterschiede und Gemeinsamkeiten der teilnehmenden Generationen zu verhandeln, womit der Altersdiskurs zu einem expliziten Thema wurde (Franz 2012). Wenn durch konkrete Übungen in den Angeboten dazu angeleitet wurde, über das Altern zu diskutieren, entstand eine Art ,age-awareness“ - diese bot die Möglichkeit, das höhere Lebensalter als Raum von Gestaltungsmöglichkeiten zu sehen, benachteiligende Prozesse im Alter zu erkennen und diesen als Individuum entgegenzutreten.

Drittens bot die Betonung von Kreativität in den Angeboten kultureller Bildung ein Potenzial, das Alter(n) kritisch zu hinterfragen. Kreativität wurde in den untersuchten Angeboten als Möglichkeit gesehen, die eigene Selbstbeschreibung als gestaltbar zu erleben. Im Zusammenhang mit Kreativität wurden dabei außerdem der Selbstwert und das Selbstbewusstsein der Teilnehmenden als gestärkt verstanden (Cantu und Fleuriet 2018).

\section{Fazit}

Die hier skizzierten Forschungsergebnisse zeigen, dass ein praxeologischer Blick auf das Alter(n) die impliziten Orientierungen über und Verhaltungserwartungen an ältere Menschen offenlegen kann. Alter(n) als rein biologischen Determinismus zu verstehen, greift damit in einer soziologischen Analyse zu kurz. Gleichzeitig machen die Forschungsergebnisse deutlich, dass das Alter nicht ausschließlich als individueller Gestaltungsprozess verstanden werden kann. In Analogie zu „Doing Gender" weist auch hier der „Doing Age“-Ansatz darauf hin, dass es nicht nur individuelle Orientierungen sind, die das Alter(n) heute bestimmen, sondern dass auch institutionelle Adressierungen und Stereotypisierungen die soziale Kategorie des Alters kennzeichnen.

Funding Open access funding provided by University of Vienna.

Open Access Dieser Artikel wird unter der Creative Commons Namensnennung 4.0 International Lizenz (http://creativecommons.org/licenses/by/4.0/deed.de) veröffentlicht, welche die Nutzung, Vervielfältigung, Bearbeitung, Verbreitung und Wiedergabe in jeglichem Medium und Format erlaubt, sofern Sie den/die ursprünglichen Autor(en) und die Quelle ordnungsgemäß nennen, einen Link zur Creative Commons Lizenz beifügen und angeben, ob Änderungen vorgenommen wurden. 


\section{Literatur}

Amann, Anton, und Franz Kolland. 2014. Das erzwungene Paradies des Alters? Weitere Fragen an eine Kritische Gerontologie, 2. Aufl., Wiesbaden: Springer.

Bamford, Anne. 2009. The Wow-factor. Global research compendium on the impact of the arts in education, 2. Aufl., Münster: Waxmann.

Baumann, Sabine, und Kim De Groote. 2018. Kunst- und Kulturgeragogik. In Alter(n) - Lernen - Bildung. Ein Handbuch, Hrsg. Renate Schramek, Cornelia Kricheldorff, Bernhard Schmidt-Hertha, und Julia Steinfort-Diedenhofen, 205-214. Stuttgart: Kohlhammer.

Bernard, Miriam, und Michelle Rickett. 2016. The cultural value of older people's experiences of theatermaking: a review. The Gerontologist 57(2):e1-e26. https://doi.org/10.1093/geront/gnw093.

Birg, Herwig, und Ernst J. Flöthmann. 2002. Langfristige Trends der demographischen Alterung in Deutschland. Zeitschrift für Gerontologie und Geriatrie 35(5):387-399. https://doi.org/10.1007/ s00391-002-0119-0.

Both, Göde. 2015. Praktiken kartografieren. Was bringt Clarkes Situational Analysis für Praxeografien? In Methoden einer Soziologie der Praxis, Hrsg. Franka Schäfer, Frank Hillebrandt, und Anna Dániel, 197-214. Bielefeld: transcript.

Bubolz-Lutz, Elisabeth, Eva Gösken, Cornelia Kricheldorff, und Renate Schramek. 2010. Geragogik. Bildung und Lernen im Prozess des Alterns. Das Lehrbuch. Stuttgart: Kohlhammer.

Buttler, Günter. 2003. Steigende Lebenserwartung - was verspricht die Demographie? Zeitschrift für Gerontologie und Geriatrie 36(2):90-94. https://doi.org/10.1007/s00391-003-0135-8.

Cantu, Adelita G., und Jill K. Fleuriet. 2018. "Making the Ordinary More Extraordinary". Exploring creativity as a health promotion practice among older adults in a community-based professionally taught arts program. Journal of Holistic Nursing 36(2):123-133. https://doi.org/10.1177/ 0898010117697863.

Clarke, Adele. 2012. Situationsanalyse: grounded theory nach dem postmodern turn. Wiesbaden: Springer.

COM. 2014. Report from the commission to the european parliament, the council, the european economic and social committee and the committee of the regions on the implementation, results and overall assessment of the 2012 European Year for Active Ageing and Solidarity between Generations. https:// eur-lex.europa.eu/legal-content/EN/TXT/?qid=1411128908532\&uri=COM:2014:562:FIN

Cumming, Elaine, und William Earl Henry. 1961. Growing old: the process of disengagement. New York: Basic Books.

Dallmann, Gerd, Andrea Ehlert, Karl Ermert, und Thomas Lang. 2008. „Alte Meister“: wie Ältere Kompetenzen in kultureller Bildung leben und nutzen. Wolfenbüttel: Bundesakademie für kulturelle Bildung.

Flick, Uwe. 2011. Das Episodische Interview. In Empirische Forschung und Soziale Arbeit, Hrsg. Gertrud Oelerich, Hans-Uwe Otto, 273-280. Wiesbaden: VS.

Franz, Julia. 2012. Intergenerationelle Lernarrangements in der Bildungsarbeit - empirische Befunde und theoretische Reflexionen. Bildung und Erziehung 65(3):257-274. https://doi.org/10.7788/bue.2012. 65.3.257.

Fraser, Kimberly D., Hannah M. O’Rourke, Harold Wiens, Jonathan Lai, Christine Howell, und Pamela Brett-MacLean. 2015. A scoping review of research on the arts, aging, and quality of life. The Gerontologist 55(4):719-729. https://doi.org/10.1093/geront/gnv027.

Gallistl, Vera, und Anna Wanka. 2016. Bildung im Dritten Lebensalter. Potentiale und Zugangsbarrieren der Bildung in der nachberuflichen Phase. Working Paper Series am Institut für Soziologie, Bd. 1. Wien: Institut für Soziologie.

Gallistl, Vera, Viktoria Parisot, und Anna Wanka. 2019. Learning to be old-The doings of age in older adult education. International Journal of Education and Ageing. In Druck.

Geertz, Clifford. 2007. Dichte Beschreibung: Beiträge zum Verstehen kultureller Systeme, 10. Aufl., Frankfurt a. Main: Suhrkamp.

Glaser, Barney G., und Anselm L. Strauss. 2010. Grounded Theory. Strategien qualitativer Forschung, 3. Aufl., Bern: Huber.

De Groote, Kim, und Flavia Nebauer. 2009. Die Phantasie ist ewig jung. Kulturelle Bildung im Alter. In Demografischer Wandel und Kultur: Veränderungen im Kulturangebot und der Kulturnachfrage, Hrsg. Andrea Hausmann, 177-201. Wiesbaden: VS.

Gulette, Margaret M. 2004. Aged by culture. Chicago: University of Chicago Press.

Havighurst, Robert J. 1961. Successful aging. The Gerontologist 1(1):8-13.

Jenkins, Andrew, und Tarek Mostafa. 2015. The effects of learning on wellbeing for older adults in England. Ageing and Society 35(10):2053-2070. https://doi.org/10.1017/S0144686X14000762 
Kontis, Vasilis, James E. Bennett, Colin D. Mathers, Li Guangquan, Kyle Foreman, und Majid Ezzati. 2017. Future life expectancy in 35 industrialised countries: projections with a Bayesian model ensemble. The Lancet 389(10076):1323-1335. https://doi.org/10.1016/S0140-6736(16)32381-9.

Litwin, Howard, und Kimberly J. Stoeckel. 2013. Social networks and subjective wellbeing among older Europeans: does age make a difference? Ageing \& Society 33(7):1263-1281. https://doi.org/10.1017/ S0144686X12000645.

Mayring, Philipp. 2007. Generalisierung in qualitativer Forschung. Forum Qualitative Sozialforschung 8(3), Art. 26. http://www.qualitative-research.net/index.php/fqs/article/view/291/639. Zugegriffen: 13. Dez. 2018.

Misoch, Sabine. 2015. Qualitative Interviews. Berlin: De Gruyter.

Noice, Tony, und Helga Noice. 2013. Participatory arts for older adults: a review of benefits and challenges. Gerontologist 53(1):256-257. https://doi.org/10.1093/geront/gnt138.

Reckwitz, Andreas. 2003. Grundelemente einer Theorie sozialer Praktiken. Eine sozialtheoretische Perspektive. Zeitschrift für Soziologie 32(4):282-301. https://doi.org/10.1515/zfsoz-2003-0401.

Reynolds, Jackie. 2015. Stories of creative ageing. Working with Older People 19(1):33-40.

Schatzki, Theodore. 2014. Art bundles. In Artistic practices: social interactions and cultural dynamics, Hrsg. Tasos Zembyles, 17-31. New York: Routledge.

Schramek, Renate. 2018. Geragogisches Wissen für die Bildungsberatung im Alter. In Bildungsberatung für Menschen im Alter. Grundlagen, Zielgruppen, Konzepte, Hrsg. Franz Kolland, Vera Gallistl, und Anna Wanka, 56-89. Stuttgart: Kohlhammer.

Schroeter, Klaus R. 2009. Die Normierung alternder Körper - gouvernementale Aspekte des doing age. In Die jungen Alten. Analysen einer neuen Sozialfigur, Hrsg. Silke van Dyk, Stephan Lessenich, 359-379. Frankfurt a. Main: Campus.

Statistik Austria. 2018. Bevölkerung. Bevölkerungsstand und -struktur. https://www.statistik.at/web_de/ statistiken/menschen_und_gesellschaft/bevoelkerung/index.html. Zugegriffen: 23. Okt. 2018.

Twigg, Julia. 2015. Clothing, identity, embodiment and age. In Textile-led design for the active ageing population, Hrsg. Jason L. Powell, Tony Gilbert, 13-24. Hauppauge, New York: Nova Science Publishers.

Twigg, Julia, und Wendy Martin. 2014. The challenge of cultural gerontology. Gerontologist 55(3):353-359. https://doi.org/10.1093/geront/gnu061.

Wanka, Anna. 2018. Disengagement as withdrawal from public space: Rethinking the relation between place attachment, place appropriation, and identity-building among older adults. The Gerontologist 58(1):130-139. https://doi.org/10.1093/geront/gnx081.

Witzel, Andreas. 2000. Das problemzentrierte Interview. Forum: Qualitative Sozialforschung 1(1):Art. 22: 1-9. https://www.ssoar.info/ssoar/bitstream/handle/document/563/ssoar-1985-witzel-das_ problemzentrierte_interview.pdf?sequence=1. Zugegriffen: 23. Okt. 2018.

Zembylas, Tasos. 2007. Kunst ist Kunst und vieles mehr. SWS-Rundschau 47(3):260-283.

Vera Gallistl MA, ist Universitätsassistentin am Institut für Soziologie der Universität Wien. In ihrer Arbeit beschäftigt sie sich mit einer kulturellen Analyse des Alter(n)s und untersucht Kreativität im Alter als ein Bestimmungsmoment eines aktivitätsorientierten, dritten Alters. In nationalen und internationalen Forschungsprojekten befasst sie sich mit den Themen Techniknutzung und „Digital Literacy“ älterer Menschen, Bildung im Alter sowie sozialen Ungleichheiten in der nachberuflichen Lebensphase.

Viktoria Parisot MA, ist Soziologin mit Spezialisierung in den Themenfeldern Familiensoziologie, Alter(n) und Generationen. Ihr Schwerpunkt liegt auf qualitativen und interpretativen methodischen Zugängen der Sozialforschung. Derzeit arbeitet sie als wissenschaftliche Mitarbeiterin am Institut für Soziologie der Universität Wien in Projekten zu Kulturgerontologie, kultureller Teilhabe und Bildung im Alter.

Julia Birke BA, absolviert ihren Master im Schwerpunkt „Familie, Generationen, Lebenslauf“ und arbeitet als wissenschaftliche Mitarbeiterin im Bereich Alterssoziologie am Institut für Soziologie der Universität Wien. Sie beschäftigt sich dabei schwerpunktmäßig mit qualitativer Forschung zu kultureller Bildung und Lernen im Alter. 